\title{
Angiogenesis at Different Stage of Pregnancy in Goat Placenta
}

\author{
T. Díaz ${ }^{1}$, C.I. Merkis ${ }^{2}$, D.S. Cots $^{1}$, E.G. Sanchis ${ }^{2}$, A.L. Cristofolini ${ }^{2}$, M.C. Romanini ${ }^{1}$, A. Bozzo ${ }^{1}$ and A. Rolando ${ }^{1}$ \\ 1. Cell Biology and Embryology, Animal Anatomy Department, School of Agronomy and Veterinary Medicine, National University of \\ Río Cuarto, Argentina \\ 2. Area Electron Microscopy, Animal Pathology Department, School of Agronomy and Veterinary Medicine, National University of \\ Rio Cuarto, Argentina
}

\begin{abstract}
The increase intransplacental exchange depends primarily on the great development of the placental vascular bed. The aim of this study was to evaluate the pattern of placental vascular development at day 50 and 100 of pregnancy in goats and the relationship with the vascular endothelial growth factor (VEGF) immunolocalization. The placental morphometric variables: capillary area density, capillary perimeter density, capillary number density and average capillary perimeter of the caruncular (CAR) and cotyledonary (COT) tissues were measured at day $50(\mathrm{n}=5)$ and $100(\mathrm{n}=5)$ of gestation. Also, the immunolocalization of VEGF was performed. In the CAR and COT tissues a significant increase of capillary area density and capillary perimeter density at day 100 of the gestation was observed. In the COT tissue, this was due to the increase in the capillary number density; however in the CAR tissue this was due to the increase in the capillary size. We can conclude that in both tissues there were different strategies to increase the capillary area and, thereby, the nutrients exchange. The immunolocalization of VEGF in the COT tissue showed the endothelial cells of blood vessels and trophoblastcells surrounding the villi intensely inmunostained, which would indicate the involvement of VEGF in the placental vascular development.
\end{abstract}

Key words: Angiogenesis, Goat, Placenta, VEGF.

\section{Introduction}

Placentomes are the structural units of ruminants placenta. They consist of interdigitated fetal cotyledonary (COT) and maternal caruncular (CAR) microvilli through which the exchange of nutrients and gases between the mother and fetus is performed [1]. The proper establishment of an extensive vascular network is required to support these important functions [2].

The establishment of functional maternal and fetal circulation is one of the first events that occurs during placental development; the proper establishment of a new vascular network (angiogenesis) is accompanied by a marked increase in the uterine and umbilical blood flow [3]. Similarly, the increase in the transplacental exchange due to the fetal exponential

Corresponding author: T. Díaz, research field: animal anatomy.E-mail: tdiazvet@gmail.com. growth during the last half of the gestation depends primarily on the dramatic growth of the placental vascular beds. Thus, factors that affect the placental vascular development will have a dramatic impact on fetal development and growth and thereby, will affect the neonatal survival and growth [4].

Angiogenesis is defined as the process that leads to the formation of new blood vessels from pre-existing vasculature [5]. Vascular growth in placental tissues begins early in pregnancy and continues through out gestation. Numerous studies using different morphometric methods to investigate the pattern of placental vascular development in ewes have been published [6-8]. Borowicz et al. have shown that the vascularization of the CAR tissue increased continuously from day 50 to day 140 of gestation, that vascularization of the COT tissues increased at about twice the instantaneous rate (i.e., the proportionate 
increase/day) of the CAR [6]. The growth of the CAR tissue was accompanied by a slight increase in the number of capillaries and by a large increase in the capillaries diameter, while in the COT tissue there was a large increase in the number of capillaries with a decrease in the capillaries diameter [6].

Numerous factors are involved in the angiogenic process, including the vascular endothelial growth factor (VEGF) and the placental growth factor (PlGF), both belonging to the VEGF family, considered the major regulatory family involved in placental vascular development $[3,4,9]$. The function of VEGF on vascular cells is to promote blood vessels growth and to stimulate vascular permeability [10]. VEGF and its receptors (VEGFR) have been found in the placentas of many species including pigs, sheep and humans [4, 10-12]. In humans, VEGF has been found in the syncytiotrophoblast and cytotrophoblastcells [13, 14]. In addition, in the sheep placenta it is known thatVEGF is produced and stored in the cytotrophoblast cells and has a paracrine effect on the endothelial cells, mediating the angiogenic process [10]. VEGF may have other biological functions. In human placenta, in the first trimester, VEGF inmunoreactive protein is present in the cytotrophoblastic shell, suggesting a role in the regulation of cytotrophoblast growth and differentiation. At term, when extensive angiogenesis has diminished, the sustained presence of VEGF mRNA in the syncytiotrophoblasts indicates that VEGF may be involved in the regulation of trophoblast function during late pregnancy [10].

The synepitheliochorial goat placenta, as the sheep placenta, facilitates the study of the maternal and fetal portions separately, since both remain intact until the end of the gestation [15].

In order to improve the reproductive rates and the offspring survival rates in goats production systems it is essential to study the placental vascular developmentand the growth factors that regulate this process.
The aim of the study was to evaluate the pattern of placental vascular development at day 50 and 100 of pregnancy in goats and the relationship with the vascular endothelial growth factor (VEGF) immunolocalization.To accomplish this, we developed histomorphometric methodologies and determined the cellular localization of VEGF by immunohistochemistry.

\section{Materials and Methods}

\subsection{Animals and Tissue Collection}

The experiments were performed in the School of Agronomy and Veterinary Medicine, National University of Río Cuarto (UNRC). The premises were adapted to the requirements of law 6344/96 of the Administración Nacional de Medicamentos, Alimentos y Tecnología Médica, Argentina. All the experimental procedures were reviewed and approved by the Ethics Committee of Research of the UNRC.

10 Criollas adult goats, fed on natural pastures and water ad libitum, were used. The estrus was determined by observation of signs of estrus behavior twice daily. The goats in the estrus period were placed with bucks. A diagnostic test by ultrasonography was made between days 25 and 30 after mating to confirm pregnancy. Five animals at 50 and at 100 days of gestation were euthanized. Immediately after the sacrifice, the reproductive tracts were removed and samples of 3 representative placentomes from the gravid uterine horn, close to the fetus, were obtained from each goat. It was fixed in $10 \%$ buffered formaldehyde for $24 \mathrm{~h}$ and processed according to the conventional histological technique. Alternate 4-5 $\mu \mathrm{m}$ histological sections were obtained using a Reichert-Young 2065 microtome and they were mounted.

\subsection{Histomorphometric Study of the Blood Vessels}

Histological sections of each of 3 placentomas, stained with hematoxylin-eosin were analyzed. Microphotographs of 10 random fields (in cluding 
base, apexofvilli andmaternal crypts) of each one of placentomes analyzed were taken at 200× magnification using a Canon Powershot G6 digital camera (Inc., Japan) attached to a Zeiss Axiophot light microscope (Carl Zeiss, Germany).

The morphometric variables area, perimeter and capillaries number were measured using the Axiovision Release 4.6.3 (Carl Zeiss, Germany) software. For CAR and COT, the capillary area density (CAD: whole capillary area per unit of tissue area), the capillary perimeter density (CPD: total capillary perimeter per unit of tissue area) and the capillary number density (CND: total capillaries numberper unit of tissue area) were calculated. Also, the average capillary perimeter (ACP) was calculated asa measure of the capillary size (Fig. 1).

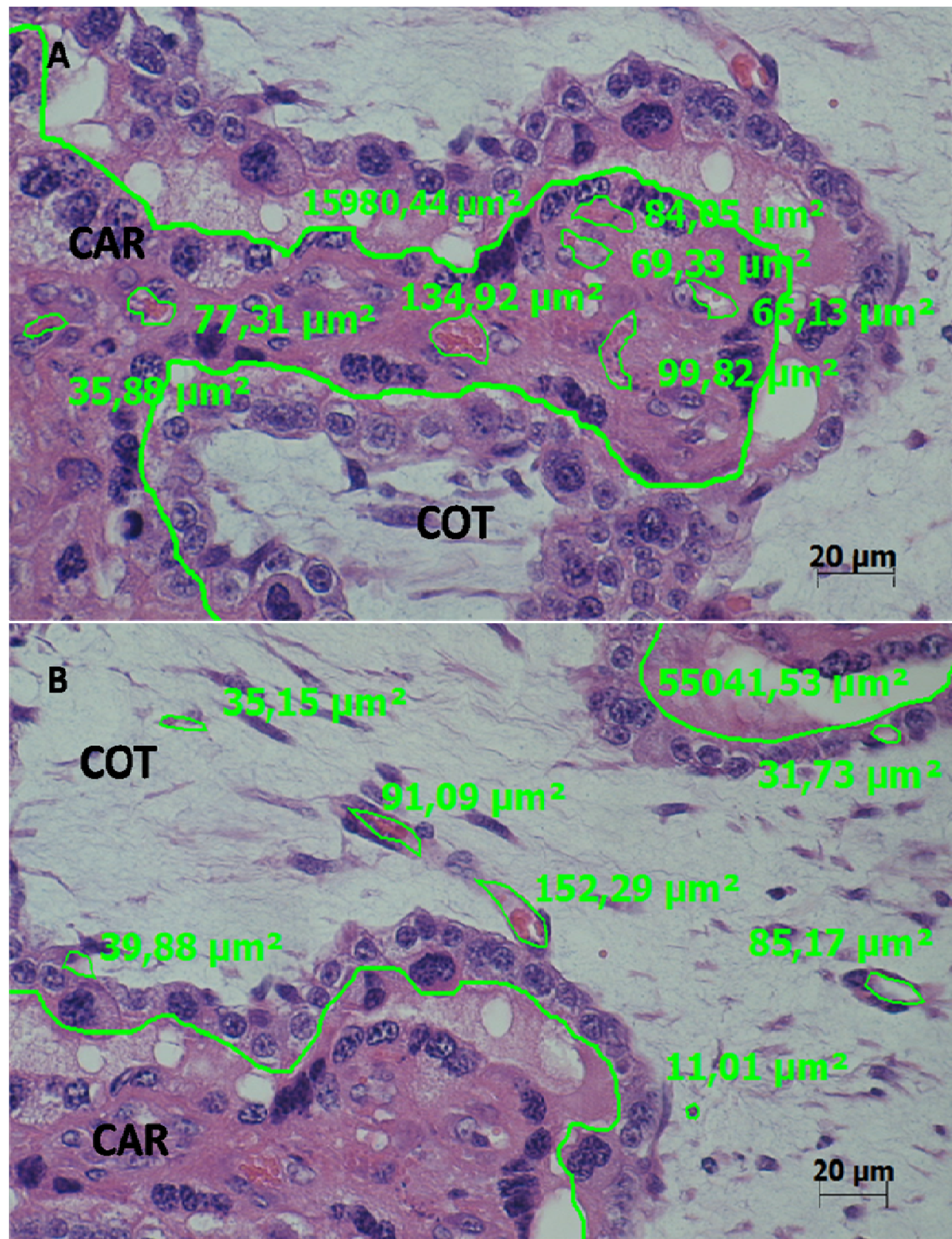

Fig. 1 Representative micrographs of placentomes at Days 100 of gestation. A: caruncular capillary area density (capillary area per unit of caruncular tissue). B: cotiledonary capillary area density (capillary area per unit of cotiledonary tissue). CAR: caruncular, COT: cotiledonary. Bar $=20 \mu \mathrm{m}$. 
A $t$-student test was performed to evaluate significant differences between groups, using the statistical software InfoStat [16]. Differences were considered significant at $P<0.05$.

\subsection{Immunohistochemistry for VEGF}

Histological sections of 2 placentomes from each placenta were analyzed. The sections were hydrated in phosphate buffered saline (PBS), $\mathrm{pH}$ 7.4. A 30\% hydrogen peroxide solution was used to block endogenous peroxidases. Nonspecific antibodies were blocked with horse serum (dilution: 1/100) for $1 \mathrm{~h}$. The sections were incubated with a commercial polyclonalanti-VEGF primary antibody (dilution 1: 100; Santa Cruz Biotechnology, USA) overnight in a wet chamber, followed by a biotinylated secondary antibody and the avidin-biotin-peroxidase complex, both for $1 \mathrm{~h}$ in a wet chamber (Vectastain ABC Elite Kit 6200; Vector laboratories, Inc., Burlingam, USA). The sections were revealed with diaminobenzidine (DAB) (Vector laboratories, Inc., Burlingam, USA) and counterstained with Mayer's haematoxylin. Microphotographs of random fields of each histological section were taken at $400 \times$ magnification using a Canon Powershot G6 digital camera (Inc., Japan) attached to a Zeiss Axiophot light microscope (Carl Zeiss, Germany). For negative control, the primary antibody was omitted.

\section{Results}

\subsection{Morphometric study of the blood vessels}

The fixation-staining methodology used allowed us to visualize and measure satisfactorily the blood vessels of the placental tissue. The capillaries maintained their normal histology and average diameter, which was similar to the one suggested by Borowicz et al. [6]. An unequal distribution of the capillaries in contact with the maternal-fetal junction zone was observed between the CAR and COTtissues.

In the COT tissue, a significant increase in the CAD
$(P<0.0001)$ and in the CPD $(P<0.0001)$ was observed at day 100 of gestation. The same pattern of behavior in the CAD $(P=0.0108)$ and the CPD $(P=$ 0.0084 ) was observed in the CAR tissue.

Comparing both regions, we observed a significant increase of the CAD in the CAR portionin relation to the COT portion at day 50 and 100 of gestation $(P=$ 0.0270 and $P=0.0013$, respectively). Moreover, a significant increase in the CPD in the CAR region in relation to the COT region at day 100 of gestation $(P$ $=0.0071$ ) was evident (Figs. 2A and 2B).

On the other hand, in the COT tissue, a significant increase in the CND at day 100 of gestation $(P<$ $0.0001)$ was observed. However, in the CAR tissue, no significant differences between the gestational stages were observed. No significant differences were observed in the CND between the CAR and the COT at day 50 or 100 of gestation (Fig. 2C).

In the CAR tissue, the ACP was significantly higher at day 100 of gestation $(P=0.001)$, whereas in the COT tissue no significant differences between gestational stages were observed. Comparing the ACP between the two regions, a significant increase in the CAR region in relation to the COT region was observed at day $50(P<0.0001)$ and $100(P<0.0001)$ of gestation (Table 1).

\subsection{Immunohistochemistry for VEGF}

VEGF was immunolocalized in both maternal and fetal tissues at the two gestational periods analyzed. In the COT tissue, the endothelial cells of the blood vessels and the trophoblastic cells around the fetal villi, were intensely immunostained, particularly the mononucleate cells and some binucleate cells. In the stromal cells of the connective tissue of the placental villi, no immunore activity was observed. In the CAR tissue, the epithelial, endothelial and stromal cells were weakly immunostained. In all the placentomes analyzed from both periods, a similar pattern of distribution and intensity of VEGF was observed. The negative control showed no staining (Fig. 3). 


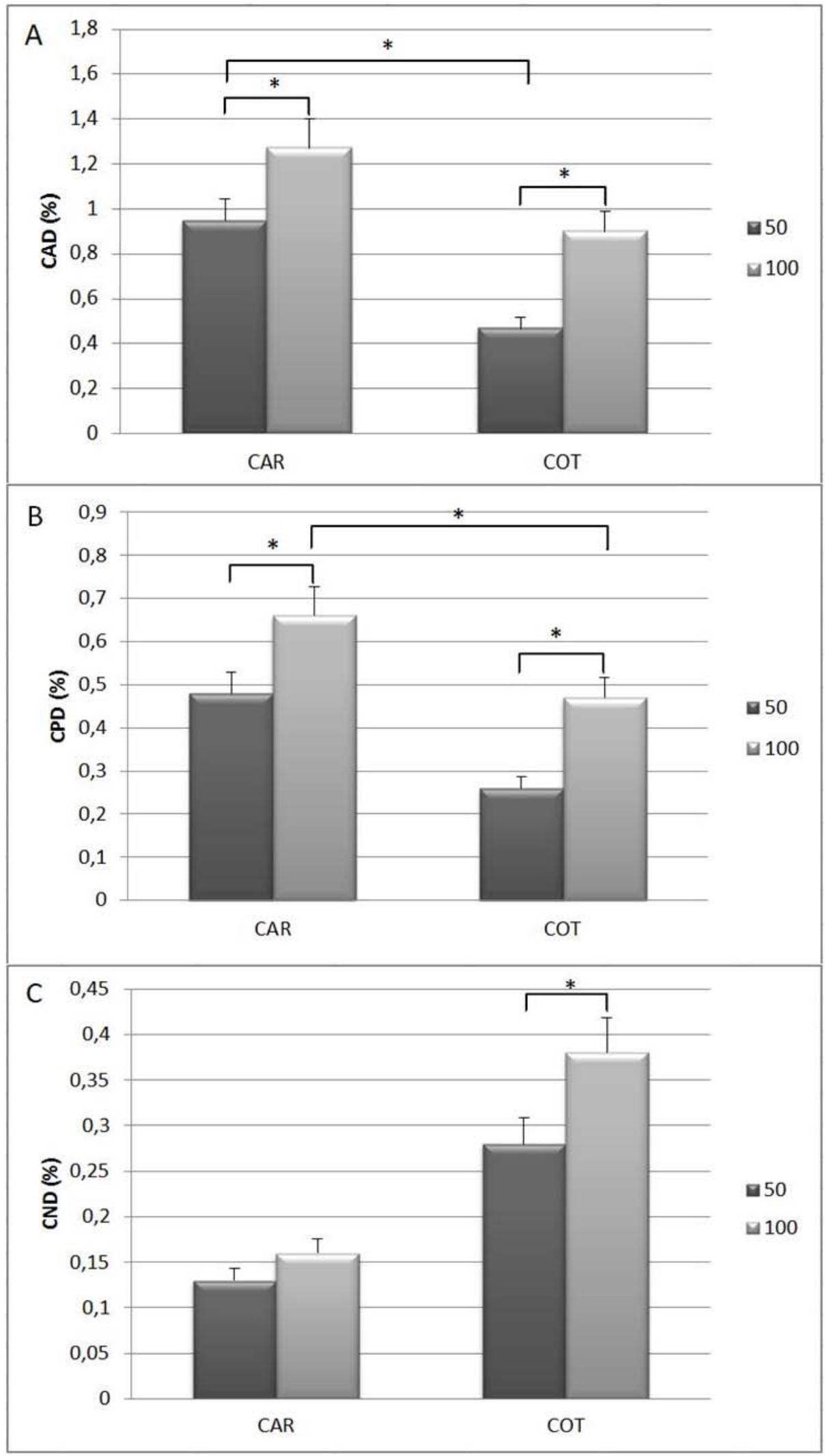

Fig. 2 A: capillary area density (CAD). B: capillary perimeter density (CPD). C: capillary number density (CND). Results are expressed in percentage $(\%)$. CAR: caruncular, COT: cotyledonary. $* P \leq 0.05$. 
Table 1 Average capillary perimeter (ACP) in CAR and COT tissue $(\mu \mathrm{m})$.

\begin{tabular}{llllll}
\hline \multicolumn{7}{c}{ Days of gestation } \\
\hline Tissue & Day 50 & SD & Day 100 & SD & $P$ value \\
\hline CAR & 38.21 & 4.19 & 41.26 & 3,80 & $0.001^{*}$ \\
COT & 31.72 & 4.54 & 33.68 & 4,24 & 0,056 \\
$P$ value & $<0.0001^{*}$ & & $<0.0001^{*}$ & & \\
\hline Da & & &
\end{tabular}

Data are expressed as mean $+/$ - standard deviation (SD).

$* P \leq 0.05$ mean statistically significant differences.

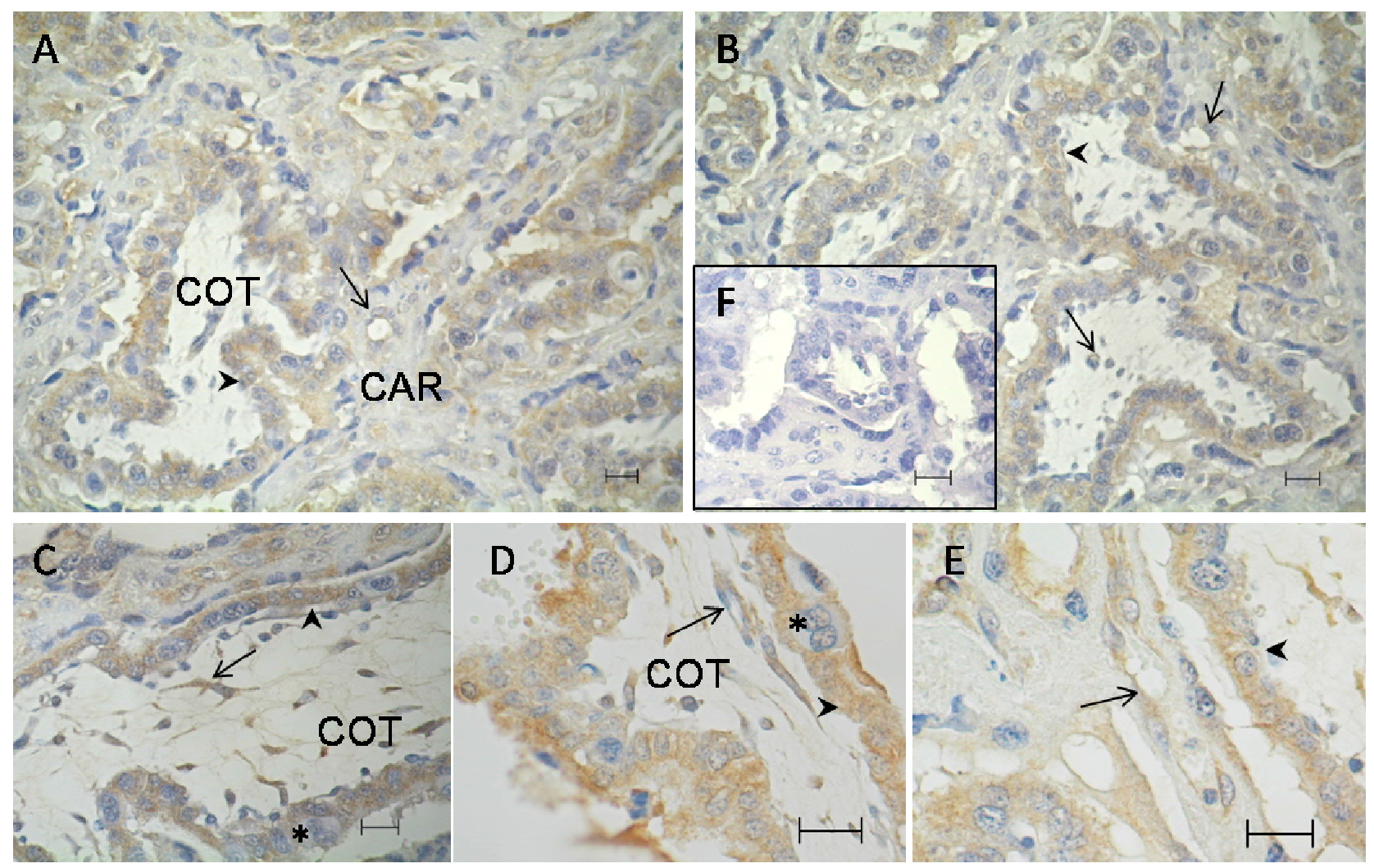

Fig. 3 Immunohistochemical localization of vascular endothelial growth factor (VEGF) in goat placenta at day 50 (A and C) and 100 (B, D and E) of gestation. F: negative control.The endothelial cells and the trophoblastic cells around the fetal villi were intensely immunostained. CAR: caruncular, COT: cotyledonary, arrowheads: trophoblastic epithelium, black arrow: endothelial cells, *: binucleate cells of the trophoblastic epithelium. Bar $=20 \mu \mathrm{m}$.

\section{Discussion}

Placental development and increase of blood flow for the maintenance of pregnancy depend on the proper vascular network formation, which arises from the vasculogenesis and angiogenesis processes, regulated both by several factors and their receptors [15]. Several studies in sheep placentas have shown that the development of placental vascular tissue in the caruncular and cotyledonary portions begins in early pregnancy and continue through out gestation [4].
In the study on goat placenta, the angiogenesis was evaluated at the beginning of the second and the third trimester of pregnancy (day 50 and 100) because several studies in sheep placenta showed that in these periods, the highest growth and differentiation of capillary network occur [6]. The vascular area density of the sheep placenta, increases slowly in the maternal tissue from mid-gestation, while in the fetal tissue itincreases sharply in the last third [6]. Consistent with these findings, in our study, a significant increase in the capillary area density at day 100 of gestation was 
observed in both, the caruncular and the cotyledonary tissues. Moreover the capillary area density of the caruncular region was greater than that of the cotiledonary region in both periods analyzed.

In the histomorphometric analysis, a significant increase in the capillary size of the caruncular tissue was observed with the advances of gestation. However, no significant differences in the capillary number density were observed, so the in creasein the vascular area may be due to an increase in the capillary size and not in the capillary number. In contrast, in the cotyledonary tissue at day 100 of gestation a significant increase in the capillary number density was observed whereas no significant differences in the average capillary perimeter were found. Thus, we can conclude that the increase in the vascular area may be due to an increase in the number and not in the size of capillaries. These results are in agreement with those observed in placentas of other species, such as sheep and humans $[6,17]$.

The umbilical and uterine blood flow increases exponentially during gestation, depending on the development of the placental vasculature [15]. In the study, the microvascular architecture observed in the cotyledonary tissue, composed of numerous small capillaries, would ensure the rapid blood transport, improving the fetal-maternal exchange $[4,15]$.

The formation of new blood vessels or new vascular network from preexisting vessels is a multistep process that is regulated by several growth factors, among which the VEGF family has been the most extensively studied $[3,4]$. In goat placentas of day 50 and 100, a strongly immunostaning of VEGF was observed in the trophoblastic epithelial and endothelial cells of the fetal blood vessels. In the maternal tissue, a weakly immunostaning of the VEGF was observed in the epithelial and endothelial cells. These results are consistent with those found in human placenta, in which VEGF was localized in the syncytiotrophoblast and cytotrophoblast cells, surrounding the fetal villi in the second and third trimester [14, 18]. Studies in human and sheep placentas suggest that VEGF expression in the trophoblast cells has a paracrine action on the endothelial cells, mediating the blood vessels growth [10]. Also, VEGF may regulate the trophoblastic proliferation, acting as an autocrine mitogenic factor and this effect could be mediated by the Flt-1 receptor of the trophoblastic cells $[13,19]$. The VEGF location found in this study, similar to that described for sheep and human placentas, may indicate that VEGF of goat placenta could have the same function [10, 13, 14]. However, further studies are needed to confirm these hypotheses.

In conclusion, in the study we observed that as the pregnancy progresses, the capillary area density in the caruncular region increases due to the growth of capillary size, while in the cotyledonary region the increase is due to the rise in the capillary number density. These changes allow the increase of blood flow, necessary to support the high demand of nutrients by the growing fetus. It is already known that the vascular growth is regulated by angiogenic factors and their receptors. In the present study carried out in goat placentas, the finding of VEGF inmunostaining in the trophoblast near small capillaries may indicate the involvement of VEGF in the vascular development.

\section{Declaration of Interest}

The authors report no conflicts of interest. The authors alone are responsible for the content and writing of this paper.

\section{References}

[1] Wooding, P., and Burton, G. 2008. Comparative Placentation: Structure, Functions and Evolution. Springer-Verlag Berlin Heidelberg.

[2] Espinosa, C. R. 2011. "Angiogénesis en la Placenta de los Animales Domésticos." Revista Veterinaria 22 (2): 131-8.

[3] Regnault, T. R. H., Vrijer, B., Galan, H. L., Davidsen, M. L., Trembler, K. A., Battaglia, F. C., Wilkening, R. B., and Anthony, R. V. 2003. "The Relationship between Transplacental $\mathrm{O}_{2}$ Diffusion and Placental Expression of 
PlGF, VEGF and Their Receptors in a Placental Insufficiency Model of Fetal Growth Restriction." Journal of Physiology 550 (2): 641-56.

[4] Reynolds, L. P., and Redmer, D. A. 2001. "Angiogenesis in the Placenta." Biology of Reproduction 64: 1033-1040.

[5] Ramazan, D., Sevala, Y., Huppertzb, B. 2007. "Vasculogenesis and Angiogenesis in the Early Human Placenta." Acta Histochemica 109: 257-65.

[6] Borowicz, P. P., Arnold, D. R., Johnson, M. L., Grazul-Bilska, A. T., Redmer, D. A., Reynolds, L. P. 2007. "Placental Growth throughout the Last Two Thirds of Pregnancy in Sheep: Vascular Development and Angiogenic Factor Expression.” Biology of Reproduction 76: 259-67.

[7] Vonnahme, K. A., Arndt, W. J., Johnson, M. L., Borowicz, P. P., Reynolds, L. P. 2008. "Effect of Morphology on Placentome Size, Vascularity, and Vasoreactivity in Late Pregnant Sheep." Biology of Reproduction 79: 976-82.

[8] Vonnahme, K. A, Arndt, W. J., Johnson, M. L., Borowicz, P. P., Reynolds, L. P., and Ford, S. 2004. "Placental Vascular Endothelial Growth Factor Receptor System mRNA Expression in Pigs Selected for Placental Efficiency.” J. Physiol. 554: 194-201.

[9] Ferrara, N., and Davis-Smyth, T. 1997. "The Biology of Vascular Endothelial Growth Factor." Endocrine Reviews 18: 4-25.

[10] Bogic, L. V., Brace, R. A., and Cheung, C. Y. 2000. "Cellular Localization of Vascular Endothelial Growth Factor in Ovine Placenta and Fetal Membranes." Placenta 21: 203-9.

[11] Sanchis, E. G., Cristofolini, A. L., and Merkis, C. I. 2012. Matriz extracelular y vascularización en la placenta porcina. OPN, Fg, colágeno y VEGF EAE, Editorial Académica Española. LAP LAMBERT Academic Publishing GmbH \& Co. KG. ISSN 978-3-659-00805-4. Saarbrücken, Alemania.

[12] Terman, B., Khandke, L., Dougher-Vermazan, M., Maglione, D., Lassam, N. J., Gospodarowicz, D., Persico,
M.G., Bohlen, P., and Eisinger, M. 1994. "VEGF Receptor Subtypes KDR and FLT1 Show Different Sensitivities to Heparin and Placenta Growth Factor." Growth Factors 11: 187-95.

[13] Ahmed, A., Li, X. F., Dunk, C., Whittle, M. J., Rushton, D.I., and Rollason, T. 1995. "Colocalization of Vascular Endothelial Growth Factor and Its Flt-1 Receptor in Human Placenta." Growth Factors12 (3): 235-43.

[14] Shiraishi, S., Nakagawa, K., Kinukawa, N., Nakano, H., and Sueishi, K. 1996. "Immunohistochemical Localization of Vascular Endothelial Growth Factor in the Human Placenta." Placenta 17: 111-21.

[15] Reynolds, L. P., Borowicz, P. P., Vonnahme, K. A., Johnson, M. L., Grazul-Bilska, A. T., Wallace, J. M., Caton, J. S., and Redmer, D. A. 2005. "Animal Models of Placental Angiogenesis.” Placenta 26: 689-708.

[16] Di Rienzo, J., Casanoves, F., Balzarini, M., Gonzalez, L., Tablada, M., and Robledo, C. 2009. InfoStat versión 2009. Grupo InfoStat, FCA, Universidad Nacional de Córdoba, Argentina.

[17] Ramsey 1982. The Placenta, Human and Animal. Praeger. New York.

[18] Valdés, G., Corthorn, J. 2011. "Review: The Angiogenic and Vasodilatory Utero-placental Network." Placenta 32: 170-175.

[19] Li, H., Gu, B., Zhang, Y., Lewis, D. F., Wang, Y. 2005. "Hypoxia-induced Increase in Soluble Flt-1 Production Correlates with Enhanced Oxidative Stress in Trophoblast Cells from the Human Placenta." Placenta 26: 210-217.

[20] Merkis, C., Cristofolini, A., Franchino, M., Sanchis, E., Moschetti, E., and Koncurat, M. 2006. Angiogénesis placentaria durante la gestación porcina. Revista Electrónica de Veterinaria REDVET ${ }^{\circledR}$, Vol. VII, $n^{\circ} 04$.

[21] Clark, D. E., Smith, S. K., Sharkey, A. M., and Charnock-Jones, D. S. 1996. "Localization of VEGF and expression of its receptors flt and KDR in human placenta throughout pregnancy." Human Reproduction 11: 1090-1098. 